\title{
Pesquisa científica: o que os concluintes do curso de Medicina pensam?
}

\author{
Scientific research: what do the 6thyear Medical students think? \\ Investigación científica: ¿qué piensan los cursos completos de Medicina?
}

\section{Resumo}

A pesquisa científica essencial para a produção de conhecimentos científicos. Com isso, o contato com a pesquisa científica permite o desenvolvimento do raciocínio crítico, promovendo impacto na aprendizagem dos estudantes. Como complemento à formação profissional, os projetos de pesquisa auxiliam no desenvolvimento pessoal, pois, ao avaliar a viabilidade da pesquisa, o graduando torna-se capaz de prever erros e sistematizar sua execução. No Brasil, a pesquisa científica, pelo Ministério da Educação (MEC) em 2010. E diante desse cenário, têm-se discutido alternativas para estimular a produção científica entre estudantes. Trata-se de um estudo transversal, descritivo e analítico, realizado por meio de questionário previamente estruturado, aplicado a estudantes concluintes do sexto ano do curso de Medicina de duas instituições do Estado do Pará, sendo uma privada e outra pública. O período de coleta do estudo foi de agosto a novembro de 2016. A coleta dos dados foi realizada pela aplicação de um questionário do tipo "Likert" validado e intitulado Escala de Atitudes Frente a Algumas Questões da Prática Médica, elaborado na Faculdade de Medicina de Ribeirão Preto da Universidade do Estado de São Paulo. Os dados foram submetidos ao teste estatístico de teste de Qui-quadrado de aderência pelo programa Bio-Estat 5. Participaram do estudo 111 estudantes concluintes do curso de medicina, número considerado suficiente para validação estatística. A partir dos dados do questionário, os acadêmicos de medicina reconhecem a importância da pesquisa científica para a formação médica, e consideram necessário conhecer os métodos utilizados em uma pesquisa médica.

Palavras-chave: Educação médica; Estudantes de ciências da saúde; Medicina baseada em evidências.

\begin{abstract}
Scientific research essential for the production of scientific knowledge. Thus, contact with scientific procedure allows the evolution of critical thinking, promoting an impact on student learning. As a complement to professional training, research projects help in personal development, as, when evaluating the feasibility of the research, the undergraduate is able to predict errors and systematize their execution. In Brazil, scientific research was carried out by the Ministry of Education (MEC) in 2010. In light of this scenario, alternatives to stimulate scientific production among students have been discussed. It is a cross-sectional, descriptive and analytical study, carried out through a previously structured questionnaire, applied to students graduating from the sixth year of the Medicine course at two institutions in the State of Pará, one private and the other public. The study collection period was from August to November 2016. Data collection was performed by applying a validated "Likert" type questionnaire entitled Scale of Attitudes to Some Issues in Medical Practice, prepared at the Faculty of Medicine of Ribeirão Preto of the State University of São Paulo.
\end{abstract}


The information were submitted to the statistical test of the Q-square test of adherence using the Bio-Estat 5 program. 111 medical graduate students participated in the work, a number considered sufficient for statistical validation. Established of the questionnaire data, medical students recognize the relevance of scientific research for medical training, and consider it importante to recognize the methods used in medical research.

Keywords: Education medical; Students health occupations; Evidence-based medicine.

\section{Resumen}

Investigación científica fundamental para la producción de conocimiento científico. Así, el contacto con la investigación científica permite el desarrollo del pensamiento crítico, promoviendo un impacto en el aprendizaje de los estudiantes. Como complemento a la formación profesional, los proyectos de investigación ayudan en el desarrollo personal, ya que, al evaluar la viabilidad de la investigación, el pregrado es capaz de predecir errores y sistematizar su ejecución. En Brasil, la investigación científica fue realizada por el Ministerio de Educación (MEC) en 2010. Ante este escenario, se han discutido alternativas para estimular la producción científica entre los estudiantes. Se trata de un estudio transversal, descriptivo y analítico, realizado a través de un cuestionario previamente estructurado, aplicado a estudiantes egresados del sexto año de la carrera de Medicina en dos instituciones del Estado de Pará, una privada y otra pública. El período de recolección del estudio fue de agosto a noviembre de 2016. La recolección de datos se realizó mediante la aplicación de un cuestionario validado tipo "Likert" titulado Escala de Actitudes hacia Algunos Temas en la Práctica Médica, elaborado en la Facultad de Medicina de Ribeirão Preto de la Universidad Estadual de São Paulo. Los datos fueron sometidos a la prueba estadística de la prueba chi-cuadrado de adherencia por el programa Bio-Estat 5. En el estudio participaron 111 estudiantes graduados de medicina, número considerado suficiente para la validación estadística. A partir de los datos del cuestionario, los estudiantes de medicina reconocen la importancia de la investigación científica para la formación médica y consideran necesario conocer los métodos utilizados en la investigación médica.

Palabras clave: Educación médica; Estudiantes del área de la salud; Medicina basada en la evidencia.

\section{Introdução}

A pesquisa científica, atualmente, é considerada um dos pilares para o desenvolvimento de novas e amplas condutas, além de fórmulas terapêuticas na medicina, e por isso é uma ferramenta primordial para o desempenho da ciência. Sendo que o termo pesquisa tem origem no latim, "perquirere que significa perquirir, buscar com cuidado, informar-se de" (Silva, 2004). E na visão da língua portuguesa, pesquisa é entendida como "ação de pesquisar, busca, investigação; trabalho científico que registra os resultados de uma investigação" (Borba, 2004; Xavier, 2014; Del-Masso, 2014).

A preocupação, no Brasil, com a pesquisa e desenvolvimento científico na área médica surgiu a partir da reforma sanitária ocorrida na década de 70, com o movimento pela redemocratização do Brasil, pois ampliou-se a proposta de mudanças no Sistema Único de Saúde, desde suas leis até seu modelo assistencial. Com isso, os modelos de atenção à saúde propostos utilizavam conhecimentos teóricos publicados em outros países e que descreviam suas experiências com as formulações impostas e posteriormente descritas (Souza, 2014).

A partir desse momento notou-se a importância de construir os próprios conteúdos curriculares que compunham a formação científica, e que propiciassem experiencias práticas e de capacitação teórica, promovendo uma atualização constante para enfrentar os desafios a prática clínica (Figueiredo, 2018; Menna, 2017).

Como exemplo fundamental da pesquisa científica, surgem os artigos científicos, principalmente, os periódicos de divulgação eletrônica, os quais são publicados rapidamente e abrangem um maior número de pessoas envolvidas na área médica, ampliando o processo de criticidade durante uma conduta médica (Menna, 2017; Soares, 2017).

Uma produção científica envolve, inicialmente, a busca de um tema que seja relevante para a comunidade científica e a realização de um projeto que seja planejado e executado de acordo com as normas adequadas, e encerre em uma discussão de resultados pertinentes e elaboração de uma conclusão do estudo (Xavier, 2014).

Entretanto, atualmente, os acadêmicos ainda encontram barreiras para a produção científica relacionadas a elevada carga horária curricular, acrescida da falta de recursos financeiros e de orientação (Figueiredo, 2018). Fatores esses, que promovem a diminuição de pessoas qualificadas para a execução de um projeto científico (Soares, 2017).

$\mathrm{Na}$ tentativa de amenizar as barreiras e dificuldades existentes, as faculdades de medicina no Brasil, estão ampliando a 
inclusão da Iniciação Científica na grade curricular (Soares, 2017). O desenvolvimento dessa habilidade ocorre a partir da inserção do Trabalho de Conclusão e Curso, que obriga o aluno a estruturar e executar um projeto científico, elaborando uma criticidade no acadêmico e contribuindo para a competência científica e atualização constante. Segundo estudos realizados em uma universidade de Norway, acadêmicos que possuem maior desenvoltura na produção de artigos científicos têm mais pontos que facilitam a sua entrada em uma residência médica (Jacobsen, 2018).

Além disso, um maior incentivo das faculdades de contribui para a continuação do engajamento do estudante de medicina após a graduação, ampliando o número de produções científicas que podem auxiliar na prática clínica de médicos e outros profissionais da saúde (Bjerkreim, 2019).

O presente estudo objetivou avaliar as perspectivas dos concluintes do Curso de Medicina sobre a pesquisa científica e identificar as atitudes dos estudantes em relação à pesquisa científica.

\section{Metodologia}

Trata-se de um estudo transversal, descritivo e analítico, com estudantes concluintes do sexto ano do curso de Medicina de duas instituições do Estado do Pará. O projeto foi submetido ao Comitê de Ética em Pesquisa, com o parecer 1.580.371, sendo a coleta de dados iniciada somente após sua aprovação. O período de coleta do estudo foi de agosto a novembro de 2016, com a aplicação de um questionário do tipo "Likert" validado e intitulado Escala de Atitudes Frente a Algumas Questões da Prática Médica, elaborado na Faculdade de Medicina de Ribeirão Preto da Universidade do Estado de São Paulo (Colares, 2002) que possui cinquenta e dois itens. Esta escala foi aplicada para a confecção da tese "Percepção dos concluintes de Medicina sobre aspectos relacionados a terminalidade da vida”. Porém, para não desprezar as outras informações, foram criadas extensões do estudo, fazendo uso para este de nove perguntas sobre APS. Os dados foram submetidos ao teste estatístico de Qui-quadrado de aderência pelo programa Bio-Estat 5 (Ayres, 2007).

\section{Resultados}

Participaram do estudo 111 estudantes concluintes do curso de medicina, sendo 55,9\% do sexo feminino (62/111; IC95\% 46,6 - 64,7) e 44,1\% do sexo masculino (49/111; IC95\% 35,3 - 53,4). A idade média dos participantes foi igual a 25,3 anos $\pm 4,0$ anos, variando entre 22 e 52 anos (IC95\% 24,6-26,1).

Em todos os itens que apresentavam juízo negativo quanto a pesquisa científica e a graduação médica; - observou-se frequência significativamente maior de respostas "totalmente em desacordo", tendo esta variado entre 31,2\% (figura 1; item 4 "Acho que no curso médico, desperdiça-se um tempo enorme tentando transformar estudantes de medicina em cientistas") a 75,5\% (Figura 1; item 2 - "Acho ingênuo pensar que o trabalho do médico pode contribuir para o desenvolvimento científico da medicina"). 
Figura 1. Distribuição dos estudantes quanto a concordância com os itens de sentido negativo no contexto da pesquisa científica e a graduação em medicina. Belém, 2019.

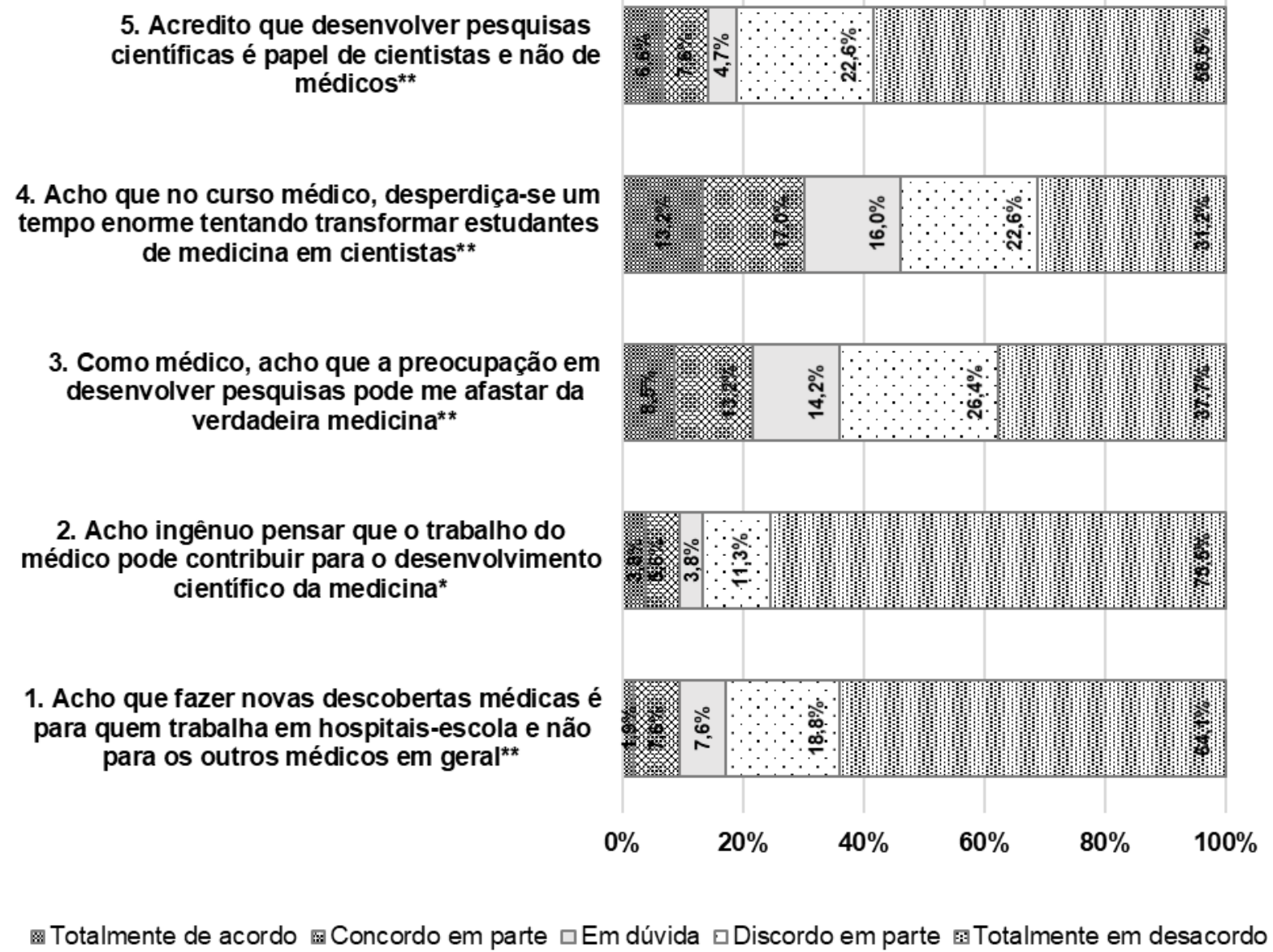

${ }^{*} \mathrm{p}<0,05 ; * * \mathrm{p}<0,01$ (Qui-Quadrado de aderência).

Fonte: Protocolo de pesquisa (2019).

No que diz respeito aos itens que dissertavam positivamente sobre a importância da ciência e do seus métodos no âmbito da graduação e do exercício da medicina, a maioria significativa dos estudantes respondeu "totalmente de acordo" a todos os itens, variando de 47,2\% (Figura 2; item 6 - "Considero importante conhecer os métodos científicos utilizados em uma pesquisa médica”) a 58,5\% (Figura 2; item 7 - “Acho importante que os estudantes de medicina, desde o início do curso, tenham o hábito de ler artigos de revistas científicas"). 
Figura 2. Distribuição dos estudantes quanto a concordância com os itens de sentido positivo no contexto da pesquisa científica e a graduação em medicina. Belém, 2019.

7. Acho importante que os estudantes de
medicina, desde o inicio do curso, tenham o
hábito de ler artigos de revistas cientificas ${ }^{\star \star}$

6. Considero importante conhecer os métodos cientificos utilizados em uma pesquisa médica**

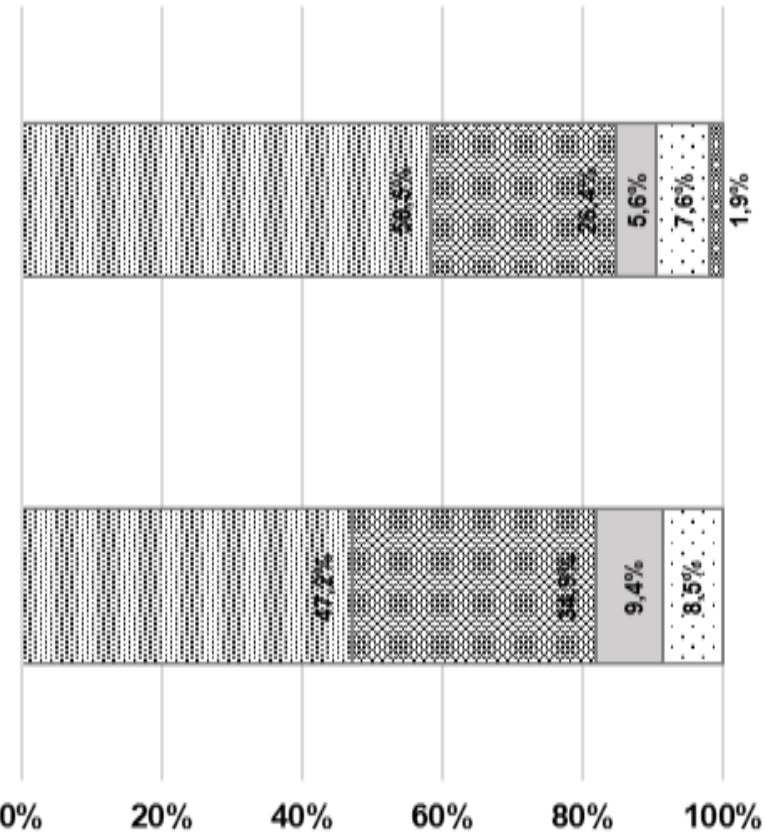

国 Totalmente de acordo 口Discordo em parte

\section{国 Concordo em parte 口Em dúvida}

**p<0,01 (Qui-Quadrado de aderência).

Fonte: protocolo de pesquisa (2019).

\section{Discussão}

Os concluintes do curso de medicina apresentam distribuição por gênero demonstrando um predomínio do sexo feminino, que correspondeu a 55,9\% dos participantes. Diversos estudos, no que tange ao gênero, apresentou resultados divergentes, tendo em vista que não apresentaram diferenças significativas entre os sexos feminino e masculino (Fiqueiredo, 2018; Soares, 2017; Oliveira, 2008). Quanto a caracterização do perfil dos alunos, os pesquisadores encontraram a média de faixa etária de 23,35 anos, similar às indicadas na literatura e descrito neste artigo (Fiqueiredo, 2018).

Este estudo obteve como resultado a ocorrência significativamente maior de atitudes positivas dos estudantes de medicina frente a pesquisa científica. Assim como, uma pesquisa realizada em seis escolas médicas de quatro estados da federação entre 2005 e 2007, a qual constatou que de 413 alunos da graduação, 56\% realizaram atividades de iniciação científica em sua faculdade e $28 \%$ não participaram de tais programas (Oliveira, 2008).

A pesquisa científica tem sido amplamente discutida nas últimas décadas, principalmente, no que tange a sua inserção na grade curricular, assim como os fatores que podem influenciar negativamente na sua produção. Segundo pesquisas, apenas $7 \%$ dos estudantes da graduação não têm interesse em participar de programas de iniciação cientifica, corroborando com o resultado exposto no presente artigo (Oliveira, 2008). Entre os fatores encontrados que influenciam negativamente na produção científica estão: em percentuais menores, a falta de interesse do aluno; e em percentuais maiores, a falta de orientação qualificada e a carência de condições materiais (Fiqueiredo, 2018; Soares, 2017; Oliveira, 2008).

Atualmente, estudos têm demonstrado o crescente interesse e envolvimento dos alunos de medicina na Iniciação Científica durante a graduação, tendo em vista o maior juízo positivo constatado em questionários aplicados. Este fator, agre ga 
uma possibilidade de melhor formação e qualificação acadêmica, tornando os graduando de medicina mais aptos a desenvolver projetos científicos (Resende, 2013).

\section{Conclusão}

É possível inferir, a partir da pesquisa realizada, que os concluintes do curso de medicina reconhecem o importante papel da pesquisa científica desde o início da graduação, criando um ambiente propicio para o planejamento, desenvolvimento e execução e projetos científicos. Os quais ampliam a criticidade do estudante de medicina e o acervo disponível para pesquisas médicas de qualidade que possam auxiliar na escolha de condutas clínicas. Ademais, percebeu-se através da pesquisa que os estudantes concluintes do curso de medicina consideram importante a implantação da Iniciação Científica na graduação, facilitando a orientação dos projetos por pessoas qualificadas.

Com base nesse desfecho, evidencia-se a importância de mais pesquisas referentes ao assunto com objetivo de expandir as discussões a respeito do tema, cuja relevância promove a maior execução de pesquisas cientificas, com consequente desenvolvimento da medicina baseada em evidências.

\section{Referências}

Alrefaie, Z., Al-Hayani, A., Hassanien, M., \& Hegazy, A. (2020). Implementing group research assignment in undergraduate medical curriculum; impact on students' performance and satisfaction. BMC Medical Education, 20 (1), 1-7.

Ayres, M., Ayres Jr, M., Ayres, D. L., \& Santos, A. A. S. (2007). Bioestat 5.0 aplicações estatísticas nas áreas das ciências biológicas e médicas. Belém: Instituto Mamirauá.

Bjerkreim, A. T., Eskerud, I., Guttormsen, A. B., \& Müller, K. E. (2019). Are participants in the Medical Student Research Programme continuing to engage in research? Tidsskrift for Den norske legeforening.

Cain, L., Kramer, G., \& Ferguson, M. (2019). The medical student summer research program at the University of Texas Medical Branch at Galveston: building research foundations. Medical education online, 24(1), 1581523.

Cardoso, G. P., Silva Júnior, C. T. D., Martinho, J. M. S. G., \& Cyrillo, R. J. T. (2004). Iniciação científica em medicina: uma questão de interesse para todas as especialidades. Pulmão RJ, 13 (1), 8-12.

Colares, M. F. A., Troncon, L. E. A., \& Figueiredo, J. F. C. (2002). Construção de um instrumento para avaliação das atitudes de estudantes de medicina frente a aspectos relevantes da prática médica. Revista Brasileira de Educação Médica, 26 (3), 194-203.

da Fonseca, J. J. S. (2002). Apostila de metodologia da pesquisa científica. João José Saraiva da Fonseca.

da Rocha, J. G., \& da Silva Rodrigues, C. K. (2017). Projeto de Iniciação Científica: uma proposta de processo. Universitas: Gestão e TI, 7(1).

Del-Masso, M. C. S., Cotta, M. A. D. C., \& Santos, M. A. P. (2012). Ética em Pesquisa Científica: conceitos e finalidades. Redefor Educação Especial e Inclusiva, Texto II. São Paulo: Unesp, 1-16.

Figueiredo, W. P. S., da Silva Nunes, T., de Moura, T. A., Lima, R. S. A., \& Tanajura, D. M. (2018). Pesquisa científica: conhecimento, atitudes e barreiras entre estudantes de medicina brasileiros. Arquivos de Ciências da Saúde, 25(3), 56-59.

Franco, C. A. G. D. S., Cubas, M. R., \& Franco, R. S. (2014). Currículo de medicina e as competências propostas pelas diretrizes curriculares. Revista Brasileira de Educação Médica, 38(2), 221-230.

Jacobsen, G. W., Ræder, H., Stien, M. H., Munthe, L. A., \& Skogen, V. (2018). Springboard to an academic career-A national medical student research program. Plos one, 13(4), e0195527.

Klowak, J., Elsharawi, R., Whyte, R., Costa, A., \& Riva, J. (2018). Predictors of medical student interest and confidence in research during medical school. Canadian medical education journal, 9(3), e4.

Menna, S. H. (2017). A pesquisa científica e a formação acadêmica. Prometheus-Journal of Philosophy, 10(24).

Negida, A. S. (2018). Egypt's Premier Medical Student Research Group: A New Model for Medical Student Research in Developing Countries. Cureus, $10(11)$.

Oliveira, N. A. D., Alves, L. A., \& Luz, M. R. (2008). Iniciação científica na graduação: o que diz o estudante de medicina? Revista Brasileira de Educação Médica, 32, 309-314.

Resende, J. C., Alves, R. B. D. S., Coutinho, M. D. S., Bragagnoli, G., \& Araújo, C. R. F. D. (2013). Importância da iniciação científica e projetos de extensão para graduação em medicina. Rev. bras. ciênc. saúde, 11-18. 
Research, Society and Development, v. 10, n. 10, e317101018927, 2021

(CC BY 4.0) | ISSN 2525-3409 | DOI: http://dx.doi.org/10.33448/rsd-v10i10.18927

Riva, J. J., Elsharawi, R., Daza, J., Toma, A., Whyte, R., Agarwal, G., \& Busse, J. W. (2019). Medical students' challenges and suggestions regarding research training: a synthesis of comments from a cross-sectional survey. Canadian medical education journal, 10(3), e91.

Soares, A. C. B., Gonçalves, T., Carreira, L. B., \& Ribeiro, T. C. S. (2017). Perfil científico de estudantes de Medicina em uma universidade pública. Scientia Medica, 27(2), 1 .

Souza, M. J. D., Sampaio, B. T. G., Ferreira, L. D. C. M., \& Nogueira, M. C. (2014). Interesse de estudantes de medicina na produção científica em saúde pública. Revista Brasileira de Educação Médica, 38, 512-518.

Xavier, L. N., de Oliveira, G. L., de Amorim Gomes, A., Machado, M. D. F. A. S., \& Eloia, S. M. C. (2014). Analisando as metodologias ativas na formação dos profissionais de saúde: uma revisão integrativa. SANARE-Revista de Políticas Públicas, 13(1). 